\title{
Electrophysiological Evidence for Temporal Overlap Among Contingent Mental Processes
}

\author{
Jeff Miller \\ University of California, San Diego
}

\author{
Steven A. Hackley \\ University of Missouri-Columbia
}

\begin{abstract}
A series of studies assessed perceptual-motor transmission of stimulus information by measuring lateralization of movement-related brain potentials in a choice reaction task with no-go trials. When stimuli varied in shape and size, lateralized potentials on no-go trials suggested that easily recognized shape information was used to initiate motor preparation and that this preparation was aborted when size analysis signified that the response should be withheld. This indicates that movement preparation can begin once partial perceptual information about a stimulus becomes available, contrary to an assumption of fully discrete models of information processing. By contrast, when stimuli varied only in size, no evidence for preliminary response preparation was obtained, contrary to an assumption of fully continuous models but consistent with asynchronous discrete coding models (Miller, 1982, 1988).
\end{abstract}

One of the most fundamental assumptions of cognitive psychology is that tasks are performed by using a sequence of distinct and contingent mental processes, or stages, such as perception, decision, and response execution (Broadbent, 1958; Donders, 1868/1969; Sternberg, 1969). A contingent series of stages is one in which the output of one stage is a prerequisite for processing by the subsequent stage; for example, response selection in a choice reaction task is logically contingent on prior recognition of the imperative stimulus. By contrast, perception of the color and form of a visual stimulus is not logically contingent, and, indeed, these processes are believed to be mediated by parallel neural pathways (Livingstone \& Hubel, 1988). The assumption of distinct, contingent stages is consistent with much behavioral evidence (e.g., Posner, 1978; Sanders, 1980), and the precise localization within the brain of a variety of complex cognitive functions (e.g., Kolb \& Whishaw, 1990; Petersen, Fox, Posner, Mintun, \& Raichle, 1988) suggests that many processing stages may be anatomically as well as functionally distinct. In addition to identifying and characterizing these processes, psychologists and neuroscientists have recently begun to examine how distinct mental processes may be assembled together to perform a complex task and how communication between such processes is effected.

A preliminary report of two of the experiments described in this article was made at the 30th annual meeting of the Society for Psychophysiological Research, October 1990, Boston, Massachusetts (Hackley \& Miller, 1990).

We wish to thank Albano Lopes for assistance with the data collection and analysis; Steven Hillyard for advice and for the loan of a polygraph; Lucero Hackley for assistance with the art work; Michael Coles for comments on the manuscript; and Marta Kutas, Jean Requin, and Allen Osman for helpful discussions.

Correspondence concerning this article should be addressed to Jeff Miller, Department of Psychology, C-009, University of California, San Diego, La Jolla, California 92093-0109, or to Steven A. Hackley, Department of Psychology, 210 McAlester Hall, University of Missouri, Columbia, Missouri 65211.
The question of communication has been especially controversial in the case of serial, contingent systems of stages, and three general models have been proposed. According to discrete transmission models (e.g., Sanders, 1980; Sternberg, 1969), contingent stages operate in strict temporal succession, with each process finishing before the next can begin. According to continuous transmission models (e.g., Eriksen \& Schultz, 1979; McClelland, 1979), on the other hand, contingent mental operations may overlap in time. Preliminary results from one stage are passed immediately to the next stage, so that the latter can begin without waiting for the former to reach completion. Finally, an intermediate position is represented by the asynchronous discrete coding model (Miller, 1982, 1988). This model assumes that, although contingent stages may overlap in time, communication is discrete for each separable code within a stimulus (e.g., color and form). In the case of perceptual output, transmission would occur in multiple discrete steps, with asynchronous communication of each codable attribute of the stimulus. In contrast with continuous models, the results of perceptual analysis of a particular attribute are not continuously available to response selection but, rather, are dispatched only after completion of that particular analysis. Consequently, the asynchronous discrete coding model predicts that if members of a stimulus set differ along a single dimension or along multiple dimensions that are integral (Garner, 1970), or are otherwise not represented by distinct codes, then perception will not overlap temporally with response selection.

Although continuous transmission models currently enjoy widespread popularity (e.g., McClelland, Rumelhart, \& the PDP Research Group, 1986), it would be premature to categorically reject discrete or intermediate models on the basis of either a priori arguments or the available data that directly address this issue. As an example of an a priori argument, it is sometimes asserted that the gross biological properties of the system are indicative of fully continuous processing: Neurons are continuously active; therefore communication among modular structures of the brain must be continuous. However, not all neurons are continuously active--some cells exhibit either thresholding or transient burst activity. Fur- 
thermore, if pattern coding is used (Uttal, 1973), persistent spike activity in projection neurons does not indicate continuous transmission any more than an uninterrupted FM carrier signal implies broadcast of an uninterrupted sequence of words or musical notes. A posteriori arguments are also undecisive. Although both psychophysiological and performance measures have provided evidence against fully discrete transmission under certain conditions (see reviews by Coles, Gratton, \& Donchin, 1988; Miller, 1988, 1991), these findings neither rule out asynchronous discrete coding models nor justify the generalization that transmission is never discrete for any pair of contingent stages for any possible task and stimulus combination. Indeed, convincing evidence for discrete transmission under certain circumstances has been obtained (e.g., Gottsdanker \& Shragg, 1985; Miller, 1985; Sanders \& Houtmans, 1985; Wijers, Mulder, Okita, Mulder, \& Scheffers, 1989; see also reviews by Miller, 1988, 1991; and van der Molen, Bashore, Halliday, \& Callaway, 1991).

In our view, the task at hand for the experimentalist is not to design a single critical study that will categorically determine whether transmission is continuous or discrete but, rather, to constrain cognitive models with empirical findings regarding interstage transmission under a variety of conditions. Put another way (Coles, de Jong, Gehring, \& Gratton, 1988), the important question is not whether communication between stages is continuous or discrete but, rather, when and why is it continuous or discrete. To address these questions, our study attempts to validate a paradigm for assessing temporal overlap among the stages of perception, response selection, and response preparation and attempts to define a contrast between one set of conditions under which overlap does occur (Experiments 2 and 3 ) and another under which it does not (Experiment 4).

In Experiments 2 and 3, stage overlap is tested for choice reactions to stimuli that differ with regard to two separable and easily nameable visual attributes-stimuli that are probably represented by two distinct mental codes (cf. Miller, 1982). The basic paradigm involves using imperative stimuli for which the two attributes are of differing discriminability so that analysis of the easily discriminable attribute (shape: $\mathrm{S}$ vs. T) is completed well before analysis of the other attribute (size: large vs. small). Subjects are required to make speeded choice reactions with the left or right hand in response to Ss and Ts of one size (go trials) but to withhold responses to letters of the other, slightly different size (no-go trials). Thus, in this task the rapidly discriminated attribute cues either the left or the right hand, whereas the more slowly discriminated attribute indicates whether the cued response should be released or withheld. If preliminary information regarding letter name or shape' is transmitted before the visual system has completed its analysis of size, then response selection and preparation can begin before it is known whether the response involving the cued hand should be emitted or not. This implies that, on no-go trials, preparation for a unimanual response could begin, only to be terminated prior to execution once size information is transmitted. By contrast, if perceptual-motor transmission occurs in a single discrete step following complete analysis of both attributes, there would be no reason for the subject to initiate unimanual response prepa- ration on no-go trials. These predictions are illustrated in Figure 1.

\section{The LRP as an Index of Selective Response Preparation}

Selective response preparation is measured with the lateralized readiness potential (LRP), an electroencephalographically recorded brain potential that is maximal in amplitude at scalp sites overlying motor cortex contralateral to the responding hand (e.g., Kutas \& Donchin, 1977, 1980; Vaughan, Costa, \& Ritter, 1968). We briefly discuss this measure and review arguments (cf. Coles, Gratton, \& Donchin, 1988) supporting its validity as an index of selective response preparation.

Movement-preceding potentials in humans were originally identified in association with two distinct classes of movement tasks. In the context of warned reaction-time tasks, movement-preceding negativity is referred to as the contingent negative variation (Walter, Cooper, Aldridge, McCallum, \& Winter, 1964), whereas in the context of uncued, self-paced movement tasks, it is termed the Bereitschafispotential or readiness potential (Kornhuber \& Deecke, 1965). More recent studies using a variety of recording methods in animals (e.g., Gemba, Sasaki, \& Tsujimoto, 1990) and humans (e.g., Rohrbaugh, Syndulko, \& Lindsley, 1976) have established that some of the same neural structures that contribute to the readiness potential also contribute to the portion of the contingent negative variation observed toward the end of the foreperiod in a speeded reaction task. Thus, on the basis of both neuroanatomical and functional similarities (reviewed by Rohrbaugh \& Gaillard, 1983), these surface-negative components may be considered at least roughly equivalent.

The idea of obtaining an index of selective response preparation by extracting the subcomponent of this negativity that reverses in left-right asymmetry according to the responding hand was first proposed by Kutas and Donchin (1980) and has subsequently been used and elaborated by numerous researchers (e.g., Coles, Gratton, \& Donchin, 1988; Smid, Mulder, \& Mulder, 1987). Following Coles and colleagues (e.g., Coles, Gratton, \& Donchin, 1988), we refer to this measure as the LRP, regardless of whether it is recorded in the context of a warned reaction-time task or a task involving uncued movements.

A number of arguments suggest that the LRP is a valid, real-time index of hand-specific motor preparation (cf. Coles, Gratton, \& Donchin, 1988).

1. LRP onset precedes movement onset: This characteristic gives face validity to the measure and, in addition, serves to distinguish preparatory processes-which logically must

\footnotetext{
1 The term shape is used to label the easily discriminable difference between Ss and Ts in order to parallel the word size. However, our results cannot determine which psychological code (e.g., low-level features, phonetic representation, or letter name) is most relevant. In point of fact, a related study that incorporated manipulations of featural similarity and upper-versus lowercase suggested that letter name might be the more relevant code (Miller, 1985).
} 


\section{Asynchronous Transmission}

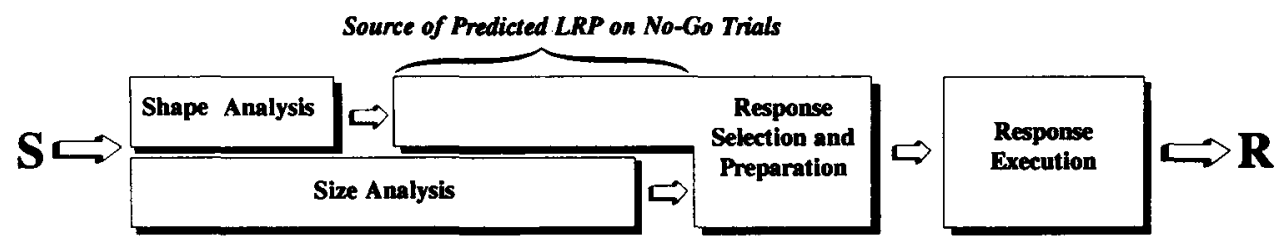

\section{Synchronous Transmission}

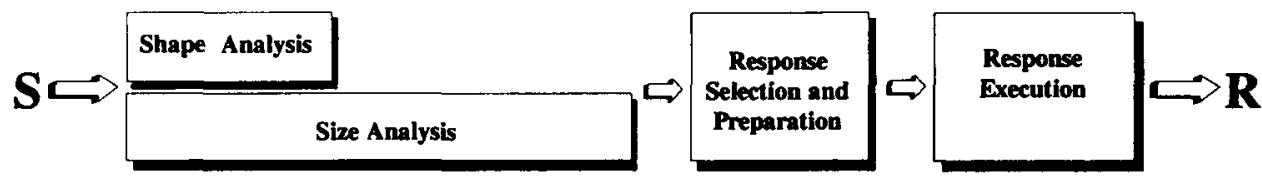

Figure 1. A schematic account of two competing models of interstage communication in human information processing. (The top panel illustrates the asynchronous discrete coding model, which assumes that perceptual-motor transmission occurs in a series of discrete pulses, one for each separable attribute of the stimulus. This model predicts that a lateralized readiness potential should be observed on no-go trials in Experiments 2 and 3. Fully continuous models of information processing would also make such a prediction. By contrast, fully discrete models, as illustrated in the bottom panel, assume that analyses of all task-relevant stimulus attributes must be completed before any perceptual-motor transmission takes place. Such models would not predict a lateralized readiness potential on no-go trials. LRP = lateralized readiness potential; $S=$ stimulus; $R=$ response.)

precede movement onset-from execution-related processes and somatosensory feedback following movement onset.

2. The LRP is generated, at least in part, by primary motor cortex: This conclusion is supported by findings obtained by using depth recording of macropotentials (e.g., Gemba et al., 1990) and single-neuron activity (e.g., Riehle \& Requin, 1989) in monkeys and by magnetic field recordings (e.g., Okada, Williamson, \& Kaufman, 1982) and voltage topographical studies (e.g., Vaughan, Costa, \& Ritter, 1968) in humans. Of special importance in emphasizing the contribution to the LRP of primary motor cortex (M1, Area 4) over other motor areas, such as premotor cortex (Lateral Area 6) and supplementary motor area (Mesial Area 6), is the phenomenon of paradoxical lateralization for foot movements (Brunia \& Vingerhoets, 1980). Recall that the motor homunculus extends over the crest of the hemispheres, with the feet and legs represented on the medial aspect of the hemispheres, within the longitudinal fissure. Because of this arrangement, if the area of $\mathrm{M} 1$ on the left hemisphere corresponding to the right ankle becomes surface negative as the subject prepares for a movement of the right foot, the current dipole will point toward the scalp electrode on the right side, the side ipsilateral to the responding limb. Consequently, the LRP generated by foot movements is of opposite polarity relative to that generated prior to hand movements. The somatotopic mapping of premotor cortex, supplementary motor area, frontal eye fields, or cerebellar cortex would not support the prediction of opposite polarity LRPs for hand versus foot movements.

3. The LRP is sensitive to certain parameters of movement: The LRP varies according to responding limb (Brunia \& Vingerhoets, 1980, as just discussed), to the complexity of movement (e.g., Hackley \& Miller, 1989), and, apparently, to the speed of movement once it is initiated (Grünewald, Grünewald-Zuberbier, Netz, Hömberg, \& Sander, 1979; but left and right hands were not compared). By contrast, the LRP is not sensitive to planned force of movement (Kutas \& Donchin, 1977) or direction of movement (Deecke, Eisinger, \& Kornhuber, 1980, cited in de Jong, Coles, Logan, \& Gratton, 1990). The fact that the LRP is sensitive to certain parameters of movement but not others supports the view that this component reflects some specific aspect of motor preparation and not, for instance, some general memory retrieval operation involved in response selection.

4. Fast guess reactions are preceded by large LRPs: Both phenomenological reports and analyses of the accuracy and latency of responses in choice reaction tasks indicate that subjects occasionally emit responses impulsively, without fully evaluating the imperative stimulus. The probability of making a particular fast guess response is predicted by the size and polarity of the LRP during the foreperiod (Gratton, Coles, Sirevaag, Eriksen, \& Donchin, 1988), thus supporting the association of this component with response-specific preparation.

5. The LRP is sensitive to response preparation cues: When a preliminary cue provides information regarding whether a left- versus right-hand response will probably be required by a subsequent imperative stimulus, an LRP of the appropriate polarity develops during the foreperiod (e.g., Gratton et al., 1990; Hackley \& Miller, 1989). The fact that LRPs can be observed during preparation for a movement that has not yet been uniquely specified (de Jong, Wierda, Mulder, \& Mulder, 1988) provides further evidence that the 
premovement LRP reflects preparatory as opposed to execution-related processes.

In summary, a number of lines of evidence provide converging support for the suggestion of Kutas and Donchin (1980) that the LRP can serve as a real-time measure of selective response preparation. The sensitivity of this component to even brief preparation for a unimanual movement is illustrated by the fact that LRPs have been observed on trials for which the response is aborted, because of a countermand stimulus, prior to muscle activation (de Jong, Coles, Logan, \& Gratton, 1990). Thus, the LRP is well suited for assessing the presence of brief, early preparation on no-go trials in the experiments we describe.

The no-go LRP paradigm used in this study was independently conceived and used by Osman, Bashore, Coles, Donchin, and Meyer $(1988,1992)$. In their study, the easy- and the difficult-to-discriminate attributes were left-right position and alphanumeric category, respectively. Consistent with the hypothesis of overlap between contingent stages, a significant LRP was observed on no-go trials, and this potential had an onset latency identical to that observed on go trials. Apparently, subjects began preparing a unimanual response as soon as left versus right target position was recognized but, on nogo trials, aborted the response (prior to muscle activation) as soon as identification of letter versus digit category indicated that the response should be withheld.

Although the same basic paradigm is used here, our experiments extend the findings of Osman and colleagues in a number of ways. First, Experiment 1 explicitly assessed the time course of perceptual analysis for the easy- and difficultto-discriminate attributes to validate a critical assumption of the paradigm, namely, that perceptual analysis finishes more quickly for the easy attribute. Second, our experiments used only stimulus attributes that are inherently nonlateralized, thereby avoiding any automatic activation of the hand ipsilateral to the reaction stimulus (Simon, 1969). Such an effect would undermine the logic of the paradigm, and special comparison conditions were required in the Osman et al. $(1988,1992)$ study to address this concern. Third, Experiment 3 incorporated control conditions, different from those used by Osman and colleagues, with the aim of ruling out certain alternative interpretations that would be consistent with fully discrete models. Convergence in our results with those of Osman and coworkers would provide strong evidence against those attempts to reconcile discrete models with evidence of preliminary response preparation. Finally, Experiment 4 examined a stimulus set for which the asynchronous discrete coding model and fully continuous models make opposing predictions, providing a test between those models not attempted by Osman and colleagues.

\section{Experiment 1: Backward Recognition Masking}

Before beginning our electrophysiological studies of interstage transmission, it was necessary to confirm that the easyand difficult-to-discriminate attributes did actually differ in the time required for their analysis. For this purpose, we chose the backward recognition masking paradigm (Massaro, 1975, pp. 355-376). There is now substantial experimental evidence that a pattern mask presented subsequent to a target stimulus impedes correct identification of the target by interrupting perceptual analysis at some point along central, modalityspecific pathways (Turvey, 1973). By varying the stimulus onset asynchrony (SOA) between target and mask, and assessing the period of vulnerability, one can obtain a rough estimate of the time course of perceptual analysis or, at least, of those analyses that precede the point at which interference occurs. Thus, the difference in the SOA at which the accuracy function reaches asymptote for letter shape versus letter size serves as our estimate of the difference in time required to perceive these attributes. Note, though, that the precise quantitative value is not critical. Rather, we merely wish to corroborate our phenomenological observations and the results of a previous reaction-time study (Miller, 1982, p. 282) indicating that shape is distinguished more rapidly than size with these stimuli.

In Experiment 1, we used the same stimulus set and response mapping as in the first electrophysiological study of the series, Experiment 2, for maximum comparability. Specifically, large and small $\mathrm{Ss}$ and $\mathrm{Ts}$ were assigned to unimanual keypresses and no-go reactions. Letter shape determined left versus right responding hand, and letter size indicated whether the response should be released or withheld. Unlike Experiment 2, the responses were unspeeded and a pattern mask was presented following letter onset in half of the blocks of trials in this study. Using these trials, perceptual accuracy was measured separately for the size and shape discriminations as a function of letter-mask onset asynchrony. In the other half of the blocks, speeded reactions were required and no mask was presented, as in Experiment 2. These blocks were included to compensate for the reduction in the opportunity for perceptual learning on masked trials. Thus, half of the blocks provided information regarding the time course of perceptual analysis, and the other half served to roughly equate perceptual learning between this preliminary study and Experiment 2.

\section{Method}

Subjects. The subjects were 60 undergraduate students, male and female, at the University of California, San Diego, who received course credit for their participation. Each subject took part in a single session lasting about $45 \mathrm{~min}$.

Apparatus. Stimuli were presented as a light-on-dark image on a NEC Multisync color monitor, Model Jc-1401P3A, controlled by an IBM-PC compatible microcomputer. The outermost three keys on the left and right sides of the bottom row of the keyboard served as manipulanda. Rather than a simple, unitary keystroke, subjects in this and the subsequent experiments were required to press a sequence of three keys, using the index, ring, and then middle fingers (i.e., the 2nd, 4th, and 3rd digits). In previous work (Hackley \& Miller, 1989), we found that such complex movements are accompanied by substantially larger LRPs than simple movements (i.e., a single keypress). The computer recorded the latency of the first keystroke of this sequence on each trial and, for each subject and trial block, generated a new random order of stimuli. Note that the longer activation time of these computer keys relative to traditional telegraph-style keys tends to produce artifactually elevated reaction times: In previous work, we observed delays of $200-400 \mathrm{~ms}$ between electromyogram onset and actual switch closure. In addition, relatively prolonged 
response preparation and execution times would be expected for complex, visually unguided finger movements (Sternberg, Monsell, Knoll, \& Wright, 1978).

Procedure. Each subject was tested in eight blocks of trials, with masked and unmasked stimuli in the even- and odd-numbered blocks, respectively. Blocks with unmasked stimuli included 16 go and 4 no-go trials, each consisting half of Ss and half of Ts. Blocks with masked stimuli were five times as long, with the preceding mixture of trials presented at each of five stimulus-mask onset asynchronies (SOAs; 75, 100, 150, 250, and $700 \mathrm{~ms}$ ). During blocks of trials with masks, accuracy was stressed; during unmasked blocks, speed was stressed.

On each trial, subjects were presented a relatively large $\left(1.0^{\circ}\right.$ in height) or small $\left(0.8^{\circ}\right)$ capital $S$ or $T$. Subjects in one group were instructed to make a left-hand response if a large $S$ was presented, a right-hand response if a large $T$ was presented, and no response if a small $S$ or $T$ was given. The letter-hand and the size-go/no-go assignments were balanced across four groups of 15 subjects. The letters $\mathrm{S}$ and $\mathrm{T}$ were equiprobable, but the go and no-go sizes occurred with relative frequencies of $80 \%$ and $20 \%$, respectively. The high proportion of go trials was essential in Experiment 2 to encourage response preparation, and Experiment 1 was intended to match that study as closely as possible. The warning stimulus was a plus sign appearing at fixation for $800 \mathrm{~ms}$, and stimulus onset occurred 500 $\mathrm{ms}$ after warning offset. The computer monitored for a response within $4 \mathrm{~s}$ following stimulus onset. Accuracy feedback was given immediately after each response was made or after the 4-s period had elapsed, and this feedback lasted $600 \mathrm{~ms}$ following correct responses and 1,200 ms following errors. The warning signal for the next trial appeared approximately $750 \mathrm{~ms}$ after the offset of the feedback for the previous trial.

The mask was a square grid of lines, approximately $4.1^{\circ}$ on a side, centered over the stimulus letter. Lines in the grid formed a crosshatching pattern, with segments oriented $45^{\circ}$ clockwise and counterclockwise from the vertical. Each line was identical in width and color to those comprising the target letters, and the parallel lines were about $0.27^{\circ}$ apart. The mask was presented from the end of the SOA until the subject responded or the end of the response period, whichever came first.

For calculation of $\mathrm{d}^{\prime}$ and $\beta$ on masked trials, one level of each stimulus dimension was arbitrarily defined as signal, the other as noise (Green \& Swets, 1966). Specifically, for calculations with regard to the shape dimension, the letter associated with left-hand reactions was considered the signal; for size analyses, the size associated with the go reaction defined a signal. Hit and false alarm rates for the shape dimension were based on go trials only, but, for the size dimension, calculations were based on both go and no-go trials. Thus, for shape, a hit was defined as a left-hand reaction to a stimulus cueing a left-hand response, regardless of whether the size of the letter indicated a go or a no-go response. If the subject responded with his or her left hand to the other shape, this constituted a false alarm. For size analyses, a hit was defined as any go response to any go stimulus regardless of shape, whereas a go response to any no-go stimulus constituted a false alarm.

\section{Results and Discussion}

Average perceptual accuracy $\left(d^{\prime}\right)$ is shown as a function of stimulus onset asynchrony in Figure 2 . The $d^{\prime}$ measure for size judgments averaged 3.90 , rose progressively across the four greatest SOAs, and even continued to improve at the longest interval used, $700 \mathrm{~ms}$, relative to the next-longest interval, $250 \mathrm{~ms}, F(1,59)=3.76, M S_{\mathrm{e}}=0.76, p<.05$, onetailed, planned comparison. By contrast, the $\mathrm{d}^{\prime}$ scores ob-

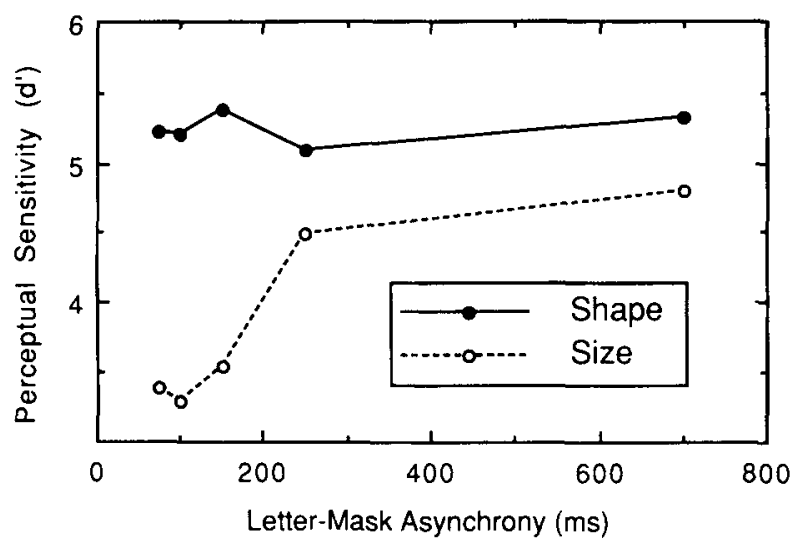

Figure 2. Average perceptual sensitivity, $\mathrm{d}^{\prime}$, for masked trials in Experiment $I$ as a function of target-mask onset asynchrony. (For unmasked trials, $\mathrm{d}^{\prime}$ averaged 5.15 for shape discrimination and 4.66 for size discrimination. The pattern of error rates mirrored these curves, with an average of $8.8 \%$ overall errors at the shortest onset asynchrony and $4.5 \%$ at the longest asynchrony.)

tained for shape judgments were higher $(M=5.25)$ and did not vary as a function of target-mask asynchrony within the $75-700 \mathrm{~ms}$ range examined, $F(4,236)=1.61, M S_{\mathrm{e}}=0.44$, n.s. Thus, analyses leading to the discrimination of the $\mathrm{S}$ versus $T$ shapes could be accurately completed in less than 75 $\mathrm{ms}$, whereas perception of the subtle difference between large and small letters required more than $250 \mathrm{~ms}$. Criterion $(\beta)$ did not vary systematically as a function of SOA for either shape or size discrimination.

The results of Experiment 1 are consistent with those of a previous experiment using reaction-time methods (Miller, 1982 , p. 282) and with one that used magnetic-pulse stimulation of visual cortex to disrupt perceptual analyses (Amassian et al., 1989). The latter study found that target-pulse onset asynchronies of less than approximately $50 \mathrm{~ms}$ or greater than approximately $130 \mathrm{~ms}$ failed to disrupt letter recognition. However, when the magnetic pulse followed the visual target by 80-100 ms, only "a blur or nothing was seen" (p. 458). The finding of perceptual invulnerability at asynchronies of less than approximately $50 \mathrm{~ms}$ is congruent with evidence from evoked potential (e.g., Hackley, Woldorff, \& Hillyard, 1990) and single-unit recording (Wilson, Babb, Halgren, \& Crandall, 1983) studies that the initial activation of primary visual cortex in humans does not begin until $30-50 \mathrm{~ms}$ after stimulus onset. The similarity between the interval of perceptual vulnerability defined by magnetic-pulse methods (80$100 \mathrm{~ms}$ ) and that defined in Experiment 1 using pattern-mask methods (asymptotic performance by $75 \mathrm{~ms}$, with an easier shape discrimination) is intriguing but must be regarded with caution, considering how little is known about the mechanisms underlying suppression in the two paradigms.

\section{Experiment 2: Early Preparation Cued by Shape}

The rationale underlying each of the currently available tests for preliminary output and stage overlap is similar. These tests require that the perceptual process be drawn out in time 
and that a qualitative difference exist between the response cued by early information as compared with the response cued by the completed perceptual analysis (Coles, Gratton, \& Donchin, 1988; Miller, 1988, 1991). Experiment 1 demonstrated that with the SSTT letter set, perceptual analysis is, in fact, drawn out in time, with completion of shape analysis preceding completion of size analysis. In Experiment 2, we used the same speeded reaction task as in Experiment 1, but, in addition, we recorded LRPs to assess whether early information about one attribute could be used to initiate response preparation before completion of analysis of the second attribute. The critical test involved the presence or absence of an LRP on no-go trials. Under the hypothesis that serial, contingent stages can overlap in time, it is expected that subjects would begin lateralized preparation of the cued response as soon as shape information is available but then, when size information becomes available, lateralized preparation would end because no response is required. ${ }^{2}$ By contrast, under the assumption that transmission of shape and size information is synchronous, no lateralized motor preparation should occur on no-go trials.

\section{Method}

Subjects. Twelve undergraduates participated in a single 3-hr session. Six of the subjects were women, and 6 were men; all were right-handed, as determined by the Edinburgh Handedness Inventory. Subjects were randomly assigned to four groups, balancing letterhand and size-go/no-go mapping, as in Experiment 1.

Apparatus and procedure. Stimulus presentation and the recording of behavioral and electrophysiological responses were carried out on the same type of computer system as in Experiment 1. A Beckman Model RM polygraph amplified the biological signals prior to digitization. The subject was seated upright in a lounge chair, with forearms resting on a cushion in front of the computer keyboard. Task and stimulus variables were identical to those described for the speededreaction blocks of Experiment 1 . The $S$ and $T$ stimuli were presented in 16 blocks of 20 trials and were preceded at an onset asynchrony of $1,500 \mathrm{~ms}$ by an 800 -ms warning stimulus, a plus sign.

Recording system. Electrophysiological responses were recorded with $\mathrm{Ag}-\mathrm{AgCl}$ electrodes attached with Grass EC-2 paste. Cerebral activity was recorded at the midparietal site, $\mathrm{Pz}$, and at $\mathrm{C}^{\prime}$ ' and $\mathrm{C4}^{\prime}$, designating sites $1 \mathrm{~cm}$ anterior and superior to the International 1020 System positions closest to the hand area of the left and right motor cortex, respectively. (For a description of these positions and an explanation of the technical terms used in this section, please consult any standard reference book on human electrophysiological methods; e.g., Cooper, Osselton, \& Shaw, 1969.) Horizontal electrooculographic (H-EOG) potentials were monitored at sites $2 \mathrm{~cm}$ lateral to the outer canthus of the left and right eyes. Electromyographic (EMG) activity from the muscles controlling finger flexion was recorded at ventral forearm sites that roughly trisected the wrist-elbow distance. Recordings of EMG potentials were made with a bipolar derivation (bandpass 0.35 to $500 \mathrm{~Hz}$ ) and were full-wave rectified offline, before averaging. All other sites were referenced to the left earlobe (A1) during data acquisition (bandpass 0.02 to $500 \mathrm{~Hz}$, with off-line digital smoothing, $11.1 \mathrm{~Hz}$ cutoff). Electrode impedance was maintained below $5 \mathrm{kOhm}$ for cephalic leads and below $15 \mathrm{kOhm}$ for EMG leads. Analog-to-digital conversion was carried out at $250 \mathrm{~Hz}$.

Signal averaging was time-locked to reaction stimulus onset, with rejection of trials having erroneous responses (including EMG on nogo trials) and trials contaminated by artifacts such as eye movements, blinking, and scalp muscular activity. Tests for artifact rejection were carried out over the window beginning $100 \mathrm{~ms}$ prior to stimulus onset and extending to the point in time corresponding to each subject's 90 th percentile for reaction time on go trials. If artifact rejection tests had included the entire 2-s recording epoch, an unacceptable number of trials would have been lost because of postresponse blinking and postural changes.

To calculate response-specific lateralization, the averaged eventrelated potentials were subjected to a two-step, millisecond-by-millisecond subtraction procedure (Gratton et al., 1988; Smid et al., 1987). First, activity at the central $\left(\mathrm{C}^{\prime}\right.$ or $\left.\mathrm{C}^{\prime}\right)$ electrode site ipsilateral to the cued hand was subtracted from activity at the contralateral site. This yielded a difference waveform equivalent to a bipolar derivation: With regard to event-related potentials for right-hand reaction trials, calculating the $\mathrm{C}^{\prime} / \mathrm{A} 1$ minus $\mathrm{C}^{\prime} / \mathrm{A} 1$ difference produces a new waveform algebraically equivalent to a bipolar $\mathrm{C} 3^{\prime} / \mathrm{C} 4^{\prime}$ derivation, where the noninverting and inverting inputs to the differential amplifier are indicated by conventional Grid-1/Grid-2 notation. In the second step, the difference waveform for trials on which the right hand was cued and the waveform for trials on which the left hand was cued were averaged together. Note that this procedure eliminates any lateralized potentials that are not response-specific (e.g., sensory evoked potentials), leaving a pure measure of movement-related lateralization (discussed further by Coles, 1989, and by Osman et al., 1992). These transformations were also applied to the ocular and EMG data to assess response-specific lateralization in these measures.

Because the readiness potential is negative at central scalp sites, lateralization that is consistent with the cued response will appear as increased negativity, with the "negative-up" convention followed in all figures. Similarly, because the ocular dipole is negative at the retina relative to the cornea, eye movements toward the cued hand also produce negative potentials in the difference waveforms. Because of rectification, all surface-recorded muscle action potentials are measured in absolute microvolts (i.e., $|\mu \mathrm{V}|$ ). To maintain consistency with presentation of the LRP and ocular data, response-specific increases in EMG activity are graphed as upward deflections. Statistical analyses were performed on both the presubtraction and the lateralization waveforms. These analyses are essentially redundant, and only results for the presubtraction analyses are reported.

\section{Results and Discussion}

Performance measures. Average reaction times decreased dramatically over the first 2 blocks of trials as subjects gained practice with the task but then were fairly stable across the subsequent 14 blocks. Therefore, the first 2 blocks were considered practice and were excluded from all subsequent analyses.

Reaction times on go trials were slightly faster for righthand than for left-hand reactions $(M \mathrm{~s}=736$ and $758 \mathrm{~ms}$, respectively), but this difference did not reach significance $(F$

\footnotetext{
${ }^{2}$ It is possible to construct models with stage overlap in which no lateralized preparation would occur, but these seem unlikely and, in fact, are inconsistent with the results of this experiment. For example, suppose that letter shape information is transmitted to response selection before size information is available, so that these two processes overlap. Suppose furthermore, however, that response selection takes much longer for letter shape than for size, so that response selection ends at the same time for the two attributes. In that case, both sources of information would become available to the response preparation stage at the same time, and no lateralized preparation would occur, despite the overlap of perception and response selection.
} 
$<1)$. Average reaction times for subjects ranged from 530 to $850 \mathrm{~ms}$, except for 1 subject whose overall mean was 1,350 ms. Error rates on go trials averaged $1.9 \%$ and were below $6 \%$ for all subjects. Errors on go trials necessarily involved making some response, because the program waited indefinitely for a keypress on these trials. About half of these errors involved hitting the incorrect key with the correct hand (i.e., keyboard errors), and the rest involved starting to make the response associated with the other letter name (i.e., hand errors). If the initial keypress was correct, the subsequent two keypresses were also correct $98.5 \%$ of the time. Movement time, measured as the interval from the first to the last keypress in the three-response sequence, averaged $390 \mathrm{~ms}$ and did not differ between hands.

The rate of incorrect responding on no-go trials (i.e., false alarms) averaged $3 \%$. Across subjects, all but one of the false alarms was a response on the side associated with the name of the stimulus letter. Thus, these false alarms were not fast guesses but were clearly based on a partial analysis of the stimulus (i.e., its shape). False alarms averaged $386 \mathrm{~ms}$ slower than correct reactions on go trials across the 12 subject-hand combinations for which false alarms occurred, $F(1,11)=$ 16.86, $M S_{\mathrm{e}}=52,982, p<.01$. Thus, false alarms apparently did not arise from aborting the perceptual process as soon as letter shape information became available but, rather, from erroneous (and slow) discrimination of letter size.

Electrophysiological measures. Figure 3 shows the grand average ( $N=12$ ) event-related potentials from which subtraction waveforms were derived. The general pattern was similar to previous findings for choice and go/no-go reaction tasks (e.g., Goodin, Aminoff, \& Shefrin, 1990). A parietally maximal P3b potential, peaking at about $300 \mathrm{~ms}$ on go trials and about $450 \mathrm{~ms}$ on the less frequent no-go trials, is evident in Figure 3 , as is a longer lasting positivity. This steady positivity, which persists to the end of the recording epoch, probably reflects a return to baseline following resolution of the contin-

\section{Go Trials}

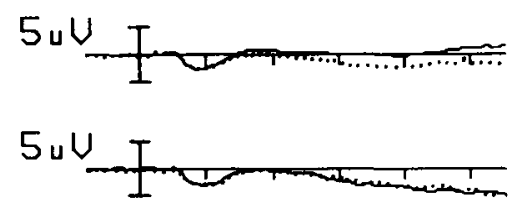

L-EOG

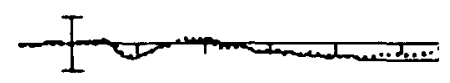

R-EOG
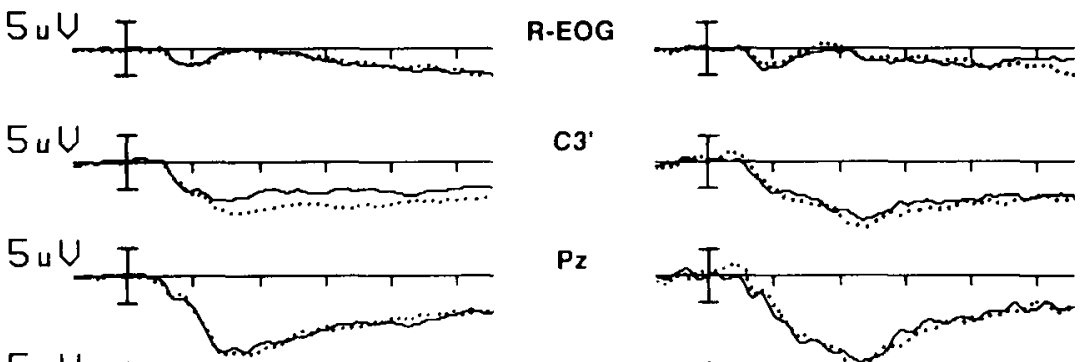

$5 . \mathrm{U}$

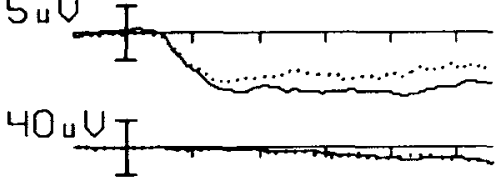

C3'

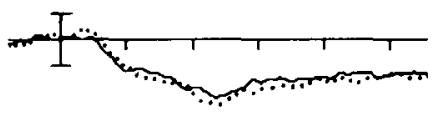

$\mathbf{P z}$

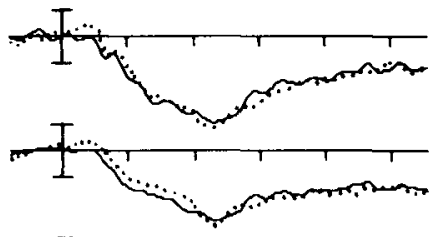

V-EOG
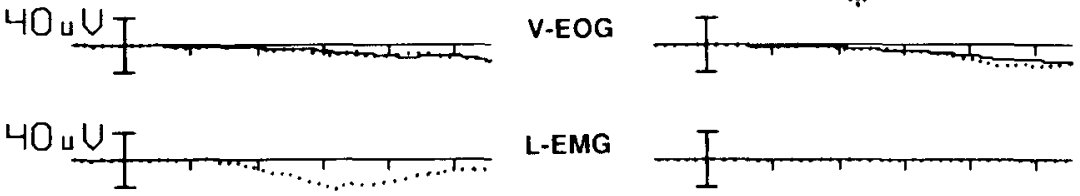

L-EMG
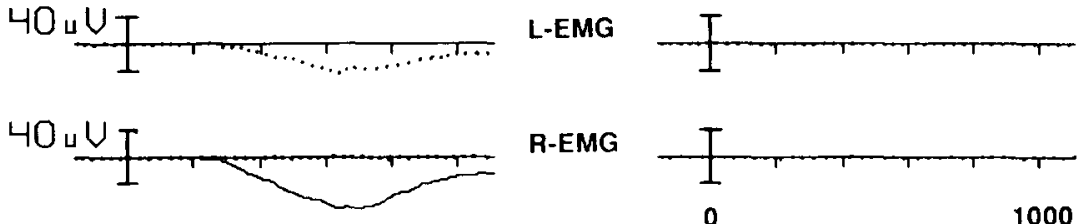

R-EMG

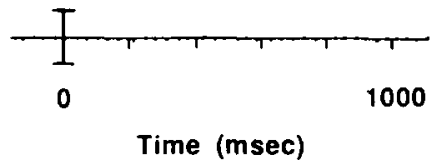

Figure 3. Grand average $(N=12)$ event-related potentials for go and no-go trials of Experiment 2 in which either the left (dotted lines) or right (solid lines) hand was cued by letter shape. (Negativity is plotted upward and, in the case of the left- and right-electro-oculogram [L-EOG and R-EOG] waveforms, indicates an eye movement away from the ocular electrode. For the vertical electro-oculogram, V-EOG, positive potentials indicate either an upward eye movement or a blink. Positive values for the rectified electromyogram, EMG, indicate an increase in activity for the forearm muscles mediating the keypress response. In these presubtraction waveforms, the lateralized readiness potential is evident as greater negativity - that is, less positivity-at the $\mathrm{C} 3^{\prime}$ electrode as compared with the $\mathrm{C}^{\prime}$ ' electrode on trials for which the right hand is cued by letter shape [solid lines], and vice versa for left-hand trials [dotted lines]. Each curve in this figure represents an average of about 1,300 responses for go trials or 330 responses for no-go trials.) 
gent negative variation induced during the warning interval (Walter et al., 1964) and, possibly, a long-lasting endogenous positivity as well (cf. Exp 4, later). Superimposed on this positivity at central sites (C3' and $\mathrm{C4}^{\prime}$ ) is an unambiguous, response-specific lateralization.

Lateralization waveforms for central, ocular, and electromyographic recordings are illustrated in Figure 4 (see also Table 1). On go trials, lateralization at central sites began at $200-250 \mathrm{~ms}$ following stimulus onset, peaked at $300-400 \mathrm{~ms}$, and continued to the end of the recording epoch. The relatively long duration of LRP is not surprising, given the time required to execute the three-part movement. (Note, though, that the portion of the LRP that follows movement onset is of limited interest because contemporary recording techniques cannot directly distinguish laterally specific efferent activity from afferent proprioceptive and cutaneous feedback.) Greater negativity at contralateral than at ipsilateral scalp sites within the $250-500 \mathrm{~ms}$ window was revealed in a significant Electrode Site (C3', C4') $\times$ Cued-Hand interaction, $F(1,11)=67.1, M S_{\mathrm{e}}=38.4, p<.001$. A main effect
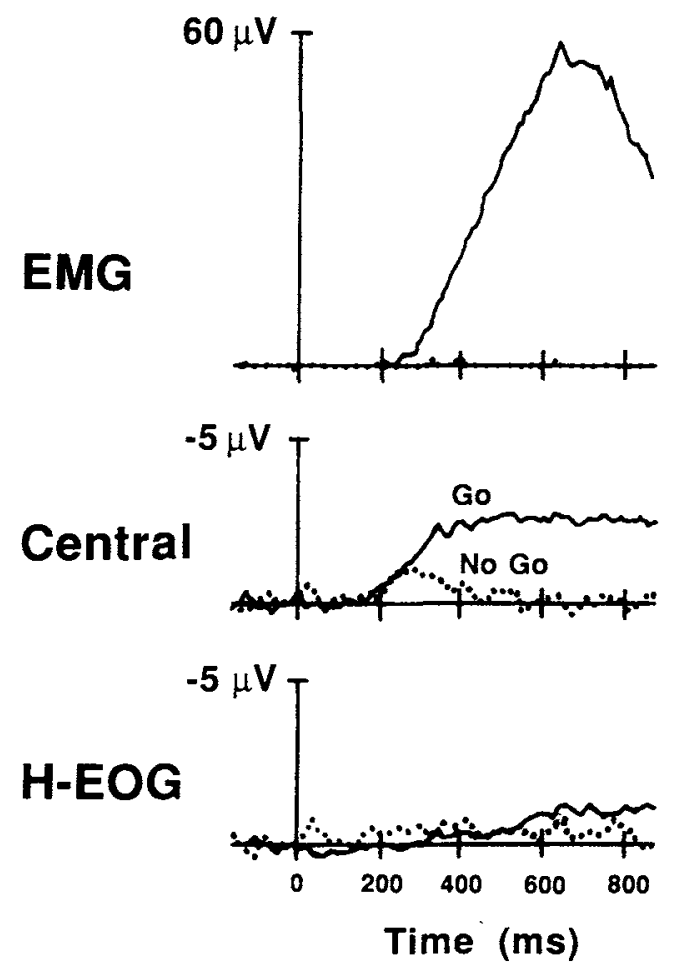

Figure 4. Grand average $(N=12)$ lateralized readiness potentials (Central), lateralized electromyograms (EMGs) and lateralized electro-oculograms (H-EOGs) on go and no-go trials of Experiment 2. (Lateralization of activity was calculated by subtracting, $\mathrm{ms}$ by $\mathrm{ms}$, the waveform for trials on which the hand ipsilateral to the noninverting electrode was cued by letter shape from the waveform for trials on which the contralateral hand was cued. Note that significant lateralization of the motor readiness potential was obtained on no-go trials [dotted lines], and this lateralization was similar in onset latency to that observed on go trials [solid lines]. Each curve in this figure represents an average of roughly 2,600 responses for go trials or 660 responses for no-go trials.)
Table 1

Mean Electrophysiological and Performance Measures for Go and No-Go Trials in Experiments 2, 3, and 4

\begin{tabular}{|c|c|c|c|}
\hline \multirow[b]{2}{*}{ Measure } & \multicolumn{3}{|c|}{ Experiment } \\
\hline & 2 & 3 & 4 \\
\hline Reaction time (ms): Go & $747.1 \pm 61$ & $671.8 \pm 31$ & $711.5 \pm 31$ \\
\hline Movement time (ms): Go & $389.7 \pm 38$ & $363.2 \pm 25$ & $419.4 \pm 39$ \\
\hline \multicolumn{4}{|c|}{ Accuracy (\% correct) } \\
\hline Go & $98.1 \pm .5$ & $98.9 \pm .4$ & $93.4 \pm .9$ \\
\hline No-go & $97.0 \pm 1.0$ & $89.9 \pm 2.3$ & $73.6 \pm 5.0$ \\
\hline \multicolumn{4}{|l|}{ LRP amplitude $(\mu \mathrm{V})$} \\
\hline Go & $1.5 \pm .2$ & $2.4 \pm .5$ & $1.0 \pm .2$ \\
\hline No-go & $.7 \pm .2$ & $1.2 \pm .4$ & $.5 \pm .3$ \\
\hline \multicolumn{4}{|l|}{ EOG amplitude $(\mu \mathrm{V})$} \\
\hline Go & $.1 \pm .1$ & $.4 \pm .2$ & $.0 \pm .1$ \\
\hline No-go & $.4 \pm .3$ & $.5 \pm .4$ & $-.2 \pm .2$ \\
\hline \multicolumn{4}{|l|}{ EMG amplitude $(|\mu \mathrm{V}|)$} \\
\hline Go & $6.8 \pm 1.8$ & $18.2 \pm 4.3$ & $6.2 \pm 1.4$ \\
\hline No-go & $.5 \pm .2$ & $1.4 \pm .3$ & $.5 \pm .2$ \\
\hline $\begin{array}{l}\text { Note. Values for elect } \\
\text { plitude of lateralization } \\
\text { (ms) and associated s } \\
\text { potential; }|\mu \mathrm{V}|=\text { absc } \\
\mathrm{EMG}=\text { electromyograr }\end{array}$ & siologica & $\begin{array}{l}\text { easures ind } \\
\mathrm{P}=\text { later }\end{array}$ & $\begin{array}{l}\text { mean an } \\
\text { nicrosecono } \\
\text { ed readine }\end{array}$ \\
\hline
\end{tabular}

due to greater negativity over the dominant hemisphere was also found, $F(1,11)=17.3, M S_{\mathrm{e}}=222.5, p<.002$.

The observed lateralization on no-go trials, shown in Figure 3 , is of greater theoretical importance. Consistent with the assumption of stage overlap, a significant LRP developed on no-go trials at about the same latency as on go trials, but then died out without producing any electromyographically indexed response activation. The analysis of variance (ANOVA) for activity at central scalp sites within the window of 250 $500 \mathrm{~ms}$ revealed a significant Site $\times$ Cued-Hand interaction and a main effect for site, $F(1,11)=13.2, M S_{\mathrm{e}}=43.9, p<$ .005 , and $F(1,11)=8.5, M S_{\mathrm{e}}=175.6, p<.02$, respectively. A combined analysis of go and no-go waveforms indicated that the LRP within this window was larger on go trials, $F(1$, 11) $=8.9, M S_{\mathrm{e}}=39.8, p<.02$. In addition, the overall greater negativity for the dominant hemisphere was more pronounced on go trials, generating a significant Site $\times$ Response (go, no-go) interaction, $F(1,11)=5.8, M S_{\mathrm{e}}=45.8, p<.05$.

For the horizontal electro-oculogram, the ANOVA revealed no significant effects for mean amplitude within the 250-500 ms scoring window. For the EMG response, measured within the same window, all main effects and interactions reached significance in the go, the no-go, and the combined analyses, except for the cued-hand effect on no-go trials. Briefly, EMG bursts were larger on go than on no-go trials, $F(1,11)=12.6$, $M S_{\mathrm{e}}=22.1$; on trials for which the right (dominant) hand was cued as compared with the left, $F(1,11)=7.8, M S_{\mathrm{e}}=$ 9.7; and at right-arm electrode sites as compared with leftarm sites, $F(1,11)=9.1, M S_{\mathrm{e}}=13.2, p \mathrm{~s}<.02$. Naturally, forearm EMG bursts were larger on the side controlling the cued hand, $\mathrm{F}(1,11)=14.7, M S_{\mathrm{e}}=21.9, p<.01$. The other interactions reflected multiplicative relations among the previously mentioned main effects, all $p \mathrm{~s}<.02$.

Although the observed LRP on no-go trials suggests that, in this task, letter shape information is used to begin response 
preparation before earlier stages are finished processing size information, an alternative interpretation must be considered, one that is consistent with fully discrete models of information processing (Miller, 1985). This interpretation assumes synchronous transmission of shape and size codes following completion of both analyses at each stage and capitalizes on the asymmetric status of these attributes at the decision level. Specifically, on no-go trials these two attributes support distinct, and mutually incompatible, decisions at the response selection stage, but only one attribute supports a lateralized response. Thus, letter shape on a no-go trial is associated with a go reaction for one hand and so tends to activate this response hand. By contrast, size information commands an inhibitory, keypress-withholding response bilaterally. These alternative reactions vie with one another until one comes to dominate, consistent with the principles of response competition first described by Sherrington (1906, lecture IV; see also Coles, Gratton, Bashore, Eriksen, \& Donchin, 1985; Stein, 1989). Hence, even on correct no-go trials, a transient period of lateralized motor preparation might be expected, resulting in a brief LRP, because of the unimanual activation provided by shape. Experiment 3 was designed to test this interpretation.

\section{Experiment 3: Effects Due to Decision-Level Asymmetry}

Experiment 3 tested the discrete interpretation of the results obtained in Experiment 2 by making a small but crucial change in the stimulus-response (S-R) mapping, such that the conjunction of size and shape had to be identified to determine whether the response should be emitted or withheld. For example, a subject might be instructed to emit the left-hand go reaction for a small $\mathrm{T}$ and the right-hand go reaction for a large $S$ and to refrain from responding for the other combinations. As a result of this change, each no-go stimulus in Experiment 3 was composed of one attribute associated with left-hand go reactions and another attribute associated with right-hand go reactions. In this example, a small $\mathrm{S}$ is a no-go stimulus. For this stimulus, therefore, the attribute small cues a left-hand go reaction (because a lefthand response is made to a small $\mathrm{T}$ ), whereas the attribute $\mathrm{S}$ cues a right-hand go reaction (because a right-hand response is made to a large $\mathbf{S}$ ).

Under the assumptions of the decision asymmetry interpretation for the no-go LRP observed in the Experiment 2, the LRP should be absent on no-go trials with this new $S-R$ assignment. On no-go trials, the decision process would receive synchronously one attribute favoring the left hand and one favoring the right hand. Thus, left- and right-hand responses would receive approximately equal activation during response selection, so there should be little or no asymmetry at motor cortex.

By contrast, if the LRP on no-go trials in Experiment 2 resulted from temporally overlapping processes, as assumed by the asynchronous discrete coding model and continuous models, then the same effect should be observed in Experiment 3. As in Experiment 2, the easily discriminable attribute, shape, still indicates which hand should be used if a response is to be emitted. Thus, response activation can begin as soon as preliminary information regarding shape is analyzed, but final response activation or inhibition is delayed because size analysis takes longer.

\section{Method}

Subjects. Twelve young adults recruited from the same pool as for Experiment 2 served as subjects. All were right-handed; 7 were women, and 5 were men. Assignment to the four Letter-Hand $x$ Size-Go/No-Go conditions was counterbalanced across subjects.

Apparatus, procedure, and recordings. The apparatus, procedure, and recording methods were the same as in Experiment 2, except for the mapping of stimuli to responses, as described earlier.

\section{Results}

Performance measures. Mean reaction times declined rapidly between the first and second blocks but remained at fairly stable levels thereafter. Hence, the first block was considered practice and was excluded from all subsequent analyses. However, movement time continued to improve slightly across the remaining 15 blocks, $F(1,14)=2.3, M S_{\mathrm{e}}=2,614.9, p<$ .01 .

The trend that was observed in Experiment 2 toward faster reactions on go trials for the right hand attained significance in Experiment 3 ( $M$ for left-hand responses $=699 \mathrm{~ms} ; M$ for right-hand responses $=645 \mathrm{~ms}), F(1,8)=8.7, M S_{\mathrm{e}}=$ $29,491.4, p<.02$. Average latencies ranged from $560 \mathrm{~ms}$ to $924 \mathrm{~ms}$ across subjects, and average error rates ranged from $0 \%$ to $3.5 \%$, with means of $672 \mathrm{~ms}$ and $1.2 \%$, respectively. Movement times were faster for the right than for the left hand $(M \mathrm{~s}=345$ and $382 \mathrm{~ms}$, respectively), $F(1,15)=18.5$, $M S_{\mathrm{e}}=6,549.3, p<.005$. There was also a group effect such that Group 4, which responded to large Ts with the left hand and small Ss with the right hand, had latencies that were slower on the average than the other three groups, $F(3,8)=$ 6.8, $M S_{\mathrm{e}}=139,833.0, p<.02$, and showed larger hand differences in movement time, $F(3,8)=5.0, M S_{e}=6,549.3$, $p<.05$. Whether this effect is related to chance differences in the assignment of subjects to groups or to the relative ease with which various stimulus-response mappings can be learned is unclear.

The rate of incorrect responding on no-go trials averaged $10.1 \%$ and ranged from $0 \%$ to $28 \%$ across subjects. The greater number of false alarms in this study relative to the preceding one (3\%) is probably due to the more complex combinatorial rule linking stimuli to responses. As in Experiment 2, though, the overwhelming majority of false alarms $(94.1 \%)$ were due to errors in size, rather than shape, discrimination. Coupled with the relatively slow average latency for false alarms $(784 \mathrm{~ms})$, this pattern of results suggests that partial stimulus information was available on these trials, but mistakes were made in analyzing size or in remembering the mapping of stimuli to responses. However, the substantial number of false alarms does not complicate the interpretation of any LRP observed on no-go trials, because no-go trials with 
responses (or even substantial EMG activity) were excluded before these LRPs were computed.

Electrophysiological measures. Both the presubtraction event-related potentials (not shown) and the difference waveforms (see Figure 5 and Table 1) were similar to those of Experiment 2. As in Experiment 2, a main effect for electrode site was obtained, reflecting greater negativity over the dominant hemisphere, $F(1,11)=6.3, M S_{\mathrm{e}}=1,058.6, p<.05$, in the combined go and no-go analysis. Similarly, lateralization of electroencephalographic activity at central sites on go trials was again observed to onset at $200-250 \mathrm{~ms}$, peak at $300-400$ $\mathrm{ms}$, and continue to the end of the recording epoch. Lateralization within the $250-500 \mathrm{~ms}$ scoring window was confirmed statistically by an interaction of electrode site and cued hand, $F(1,11)=23.7, M S_{\mathrm{c}}=296.1, p<.001$. More important, this interaction also attained significance on no-go trials, $F(1,11)$ $=8.3, M S_{\mathrm{e}}=193.1, p<.02$, although it was not as large as on go trials, as shown by an interaction of site, cued hand, and response, $F(1,11)=5.25, M S_{\mathrm{e}}=180.8, p<.05$. Thus, the presence of a brief lateralization of the readiness potential on no-go trials that was at least as large as the one recorded in Experiment 2 provides strong evidence against the discrete interpretation of the results of that study. Instead, the results support the original hypothesis, that distinct perceptual attri-

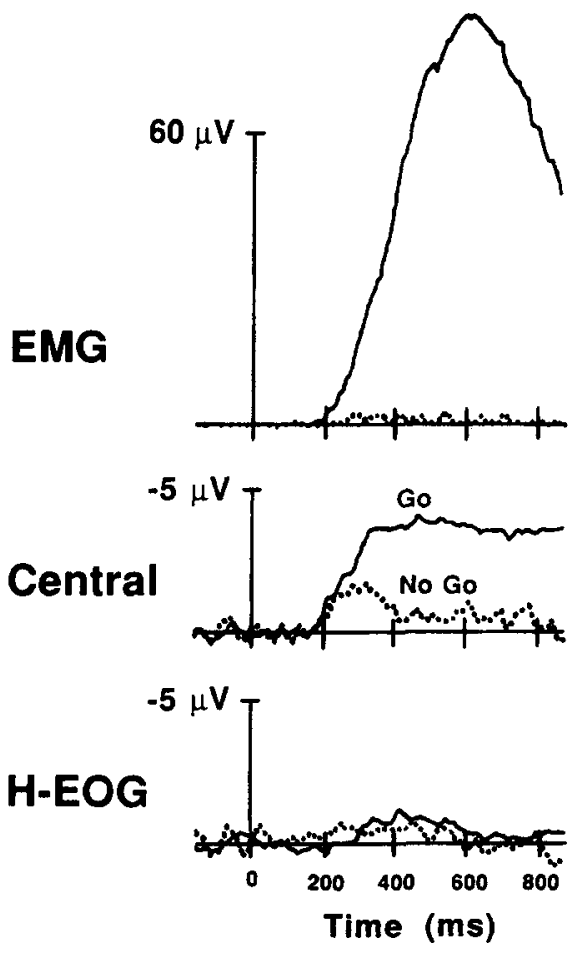

Figure 5. Grand average $(N=11)$ lateralized readiness potentials (central), lateralized electromyograms (EMGs), and lateralized electro-oculograms (H-EOGs) on go and no-go trials of Experiment 3. (Note the similarity of these results to those of Experiment 2 [shown in Figure 4]: Significant lateralization of the motor readiness potential was again obtained on no-go trials [dotted lines], and this lateralization was similar in onset latency to that observed on go trials [solid lines].) butes are asynchronously transmitted to response mechanisms as soon as they are available.

The electro-oculographic activity in Experiment 3, unlike that observed in Experiment 2, displayed significant lateralization within the $250-500 \mathrm{~ms}$ scoring window on go trials, $F(1,11)=5.1, M S_{\mathrm{e}}=32.2, p<.05$. Lateralization of EOG potentials are of special concern in readiness potential studies because lateralized gaze shifts can produce an artifact that mimics the LRP. If the subject looks toward the cued hand on an appreciable number of trials, this will generate lateralized negativity at central electrode sites because the back of the eye is negative relative to the front of the eye. To further complicate matters, large LRPs can sometimes spread as far anteriorly as the peri-ocular region, making it difficult to discern whether lateralized electro-oculographic negativity is of cerebral or retinal origin. However, the gradient of voltage distribution makes it clear that the LRPs of Experiment 3 were not artifactually produced by eye movements. According to topographical data from Hillyard and Galambos (1970), if lateralized negativity at $\mathrm{C}^{\prime}{ }^{\prime}$ and $\mathrm{C}^{\prime}{ }^{\prime}$ were actually due to contamination by ocular potentials, it should have been four to five times larger at ocular than at central sites. Instead, the lateralized negativity was larger at central sites. Furthermore, lateralization of the EOG did not attain statistical significance on the theoretically critical no-go trials, $F(1,11)=1.21, M S_{\mathrm{c}}$ $=218.2, p<.29$. These findings, together with the absence of EOG lateralization in Experiment 2, render EOG artifacts highly unlikely as an explanation of our results.

Electromyographic responses within the $250-500 \mathrm{~ms}$ window showed the same pattern of results as in Experiment 3, except that the previously observed main effect for forearm site on no-go trials (right greater than left) did not reach significance.

In Experiment 3, we attempted to rule out an interpretation of the no-go LRP that is consistent with discrete models by demonstrating LRPs on no-go trials for which one attribute was associated with left-hand go reactions and the other with right-hand go reactions. In a parallel study by Osman and colleagues (1992), a different strategy was used to arrive at the same conclusion. In Experiment 1 of that study, the difficulty of the alphanumeric discrimination determining go versus no-go was manipulated (easy was V vs. 5; hard was lowercase letter $l$ vs. number 1 ), whereas the perceptual difficulty of the attribute controlling response hand was held constant (left vs. right hemifield of presentation). Suppose, as the discrete interpretation maintains, that both attributes are synchronously transmitted to the response preparation stage as soon as analysis of the more difficult attribute is finished and that subjects cannot resist the impulse to at least partially activate a response on no-go trials. If so, an LRP would be generated on no-go trials, but its onset latency ought to be later on trials with the more difficult and time-consuming alphanumeric discrimination. It was not. Instead, onset latencies were found to be equivalent across these conditions, consistent with the assumption that differential engagement of the two hands can begin as soon as the attribute determining response hand is transmitted. The converging evidence from our research and that of Osman and colleagues clearly refutes the discrete interpretation and supports the validity of 
the no-go LRP paradigm for assessing early perceptual-motor transmission. ${ }^{3}$ The next step is to apply this paradigm to different stimulus sets, to aid in determining the circumstances under which stage overlap is possible.

\section{Experiment 4: Single-Attribute Stimulus Sets}

The results of Experiments 2 and 3 are consistent with recent evidence (reviewed by Coles, Gratton, \& Donchin, 1988, and Miller, 1988, 1991) supporting the existence of stage overlap, a possibility denied by fully discrete models of information processing, such as those of Sternberg (1969) and Sanders (1980). As described in the Introduction, the existence of stage overlap is affirmed by fully continuous models (e.g., Eriksen \& Schultz, 1979; McClelland, 1979) and by a model of information processing that is intermediate between these extremes, the asynchronous discrete coding model (Miller, 1982, 1988). Although the latter two classes of models agree that early communication is possible, continuous models assert that such communication always takes place, whereas the asynchronous discrete coding model maintains that it only occurs when the stimulus is represented by more than one distinct code (e.g., size and shape). Consequently, the theories make opposite predictions for perceptual-motor transmission in a reaction task requiring discriminations only along a single dimension.

In Experiment 4, we attempted to determine whether nogo LRPs would be observed in this paradigm if the stimuli differed with regard to a single attribute-size. The stimuli were outline squares presented at fixation, and the four possible sizes were approximately scaled according to the values $8,10,16$, and 19. To illustrate the rationale, consider a subject for whom Size 8 indicates a left-hand reaction, Size 19 a righthand reaction, and the intermediate sizes, 10 and 16 , a no-go response. Because coarse variations in size are easily distinguished, the difference between the relatively small squares ( 8 and 10 ) as compared with the relatively large ones (16 and 19 ) is presumed to be rapidly discriminated. This difference is the cue for left- versus right-hand reactions, analogous to letter shape in Experiment 2. By contrast, the difference between the two small sizes and between the two large sizes would presumably take longer to discriminate. Thus, intermediate (10 and 16) versus extreme (8 and 19) is analogous to the difficult-to-discriminate attribute of letter size in Experiment 3 , and it determines whether the reaction should be withheld (no-go trials) or emitted (go trials). To facilitate discussion, the small-large and intermediate-extreme variations are referred to as easy and hard subattributes, respectively.

Under the assumptions of fully continuous transmission, a brief lateralization should be observed on no-go trials during the period after a coarse analysis of size has finished but before the build-up of information is sufficient to distinguish the intermediate from the extreme sizes. By contrast, the asynchronous discrete coding model assumes that information about size is transmitted in a single discrete code as soon as this analysis is completed. Because this code indicates that the response should be withheld on no-go trials, no LRP should be observed for these trials.
If an LRP were observed, of course, one could always attempt to save the asynchronous discrete coding model by suggesting that multiple distinct codes were used in the transmission of information about size. As discussed by Miller (1983), however, this move would be theoretically very unappealing in the absence of independent evidence of multiple codes because it would create a circular relationship between theory and data.

\section{Pilot Study}

Before beginning the electrophysiological study, we conducted a preliminary experiment, using only performance measures, to validate our assumption that coarse variations in size could be distinguished more rapidly than fine variations. Seventy undergraduate subjects were randomly assigned to two groups, of which one received the same stimulus set as in Experiment 4 (squares), and the other the same set as in Experiment 2 (Ss and Ts). In half of the balanced blocks of trials, the subjects performed a two-choice reaction to the easy attribute (S vs. T shape) or subattribute (large vs. small squares), while ignoring the hard attribute or subattribute. During the other blocks, the subject executed the same fourchoice reaction with no-go trials as in the parallel electrophysiological study (i.e., Experiment 2 or 4). Consistent with the masking data of Experiment 1 , mean reaction time to Ss and Ts was $160 \mathrm{~ms}$ faster in the two-choice condition as compared with the four-choice condition in which letter size as well as shape had to be discriminated $(M s=451$ and 611 $\mathrm{ms}$, respectively). Similarly, reactions for subjects in the squares group averaged $158 \mathrm{~ms}$ faster in the two-choice as compared with the four-choice condition $(M s=493$ and 651 $\mathrm{ms}$, respectively). Although increases in latency across tasks may also reflect increased demands at postperceptual stages, the pattern of results does suggest that the extra time required for the intermediate/extreme discrimination with squares was comparable to the extra time required for the size discrimination with Ss and Ts. This parallel is supported by the absence of a significant Group $\times$ Task interaction, $F(1,68)<$ $1, p>.20$. We now turn to the main study of Experiment 4 .

\footnotetext{
${ }^{3}$ Note that in a previous experiment using essentially the same stimulus set, no evidence for incipient response preparation was found for no-go letters (Miller, 1985, Experiment 2). In this study, probereaction time rather than electrophysiological methods was used to assess response preparation. Specifically, a tone was presented at various onset asynchronies relative to the no-go letter, and the pitch of this tone commanded either a left- or a right-hand reaction. With other stimulus sets, early motor preparation was detected by observing faster reactions when the probe reaction was consistent, as opposed to inconsistent, with the hand designated by the easily discriminable attribute of the visual stimulus. However, no such effect was found for the stimulus set in question. The sharp contrast between the Miller (1985) results and those of our present study is difficult to explain. It appears that the probe-reaction time procedure used by Miller (1985) is less sensitive to response preparation than is the present psychophysiological procedure, but further research will be needed to determine the conditions under which probe RTs fail to reveal actual response preparation. For our purposes, the important point is that there is response preparation in this task, even if it is not measurable by some methods.
} 


\section{Method}

Subjects. Twelve undergraduate volunteers participated in the single-session experiment; all were right-handed, 8 were female. Subjects were randomly assigned to two groups balancing the mapping of small- and large-size squares onto left- and right-hand reactions. One subject failed to exhibit lateralization of the readiness potential on go trials and so, to more accurately portray LRP morphology, his data were not included for computation of the grand average waveforms. Results of statistical analyses differed little as a function of whether this subject was or was not included. The values given here are for the inclusive analyses.

Apparatus, procedure, and recordings. Stimuli were presented as light-on-dark outline images on the NEC color monitor described earlier. The squares were presented at fixation and had sides measuring approximately $0.8^{\circ}, 1.0^{\circ}, 1.6^{\circ}$, or $1.9^{\circ}$. All other aspects of the procedure were identical to those of Experiments 2 and 3 .

\section{Results and Discussion}

Performance measures. Average reaction times decreased sharply over the first three blocks of trials but were relatively stable thereafter. Consequently, the first three blocks were considered practice and were excluded from all subsequent analyses.

On go trials, reaction time averaged $712 \mathrm{~ms}$, error rate $6.6 \%$, and movement time $419 \mathrm{~ms}$. The only significant finding was a modest interaction of Group $\times$ Block $\times$ Cued Hand for response latency, $F(12,120)=2.0, M S_{\mathrm{e}}=6,405.5$, $p<.05$. Reaction times were slightly elevated at the beginning of the experiment for the responding hand designated by the relatively small sizes--the right hand for Group 1 and the left hand for Group 2. This effect may have been due to a slight imbalance in the relative difficulty of the discrimination between the two small squares as compared with the two large ones.

Error rates averaged $26.4 \%$ on no-go trials, and virtually all (>99\%) false alarms were made with the response hand assigned to the go stimulus that was similar in size to the presented no-go stimulus. The higher proportion of false alarms as compared with Experiments $2(3.0 \%)$ and $3(10.1 \%)$ indicates that the go/no-go variable was even more difficult to discriminate in Experiment 4. Consistent with the latency effect observed on go trials, errors were especially frequent for the responding hand designated by the relatively small sizes, Group $\times$ Cued Hand, $F(1,10)=19.2, M S_{\mathrm{c}}=841.3, p<$ .001 .

Electrophysiological measures. The presubtraction eventrelated potentials were similar to those of Experiments 2 and 3 and, hence, are not illustrated. Difference waveforms may be seen in Figure 6 (cf. Table 1). For go trials, LRPs were similar in latency and morphology to those observed in Experiments 2 and 3, but amplitudes were reduced for both cerebral and myogenic recordings. The finding of less forceful responses in conjunction with relatively high error rates suggests that subject confidence levels were somewhat reduced in this task. For no-go trials, lateralization at central sites differed in both morphology and amplitude, compared with that observed in the earlier experiments, and was inconsistent across subjects. The grand average waveform shown in Figure
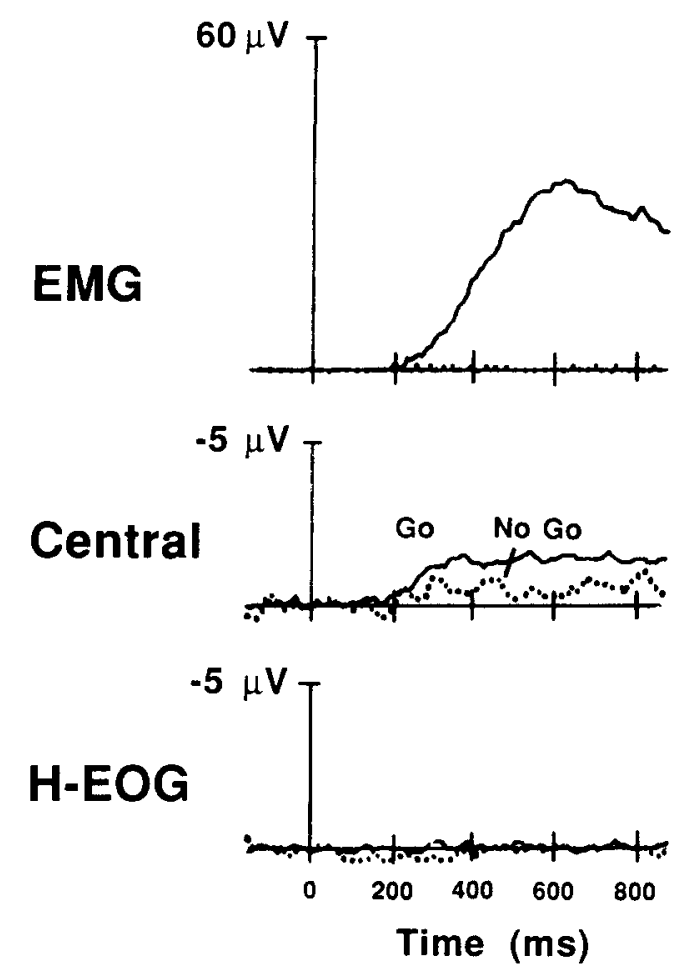

Figure 6. Grand average $(N=12)$ lateralized readiness potentials (central), lateralized electromyograms (EMGs), and lateralized electro-oculograms (H-EOGs) on go and no-go trials of Experiment 4. (In contrast with the results of Experiments 2 and 3, no brief lateralization of the readiness potential was seen on no-go trials [dotted lines]. A reliable lateralization was observed on go trials [solid lines].)

6 suggests a prolonged, low-amplitude LRP. To assess the reliability of this potential, statistical analyses were performed on the mean amplitude measured at consecutive $200-\mathrm{ms}$ epochs, for both go and no-go trials. By the second poststimulus epoch, the amplitude of the LRP reached significance on go trials, $F(1,11)=27.9, M S_{\mathrm{e}}=16.7, p<.001$, and remained so until the end of the recording epoch (all $p s<.02$ ). By contrast, the LRP on no-go trials did not attain significance at any point during the recording epoch.

A number of other electrophysiological findings are noted. Greater positivity, or reduced negativity, was observed on go trials as compared with no-go trials at every $200-\mathrm{ms}$ epoch except the first and fourth ( $p s<.05$ ). This go versus no-go difference was larger over the right than over the left hemisphere at each of the epochs from the third through the last ( $p s<.01$ ). The basis for this go versus no-go difference is unclear. As discussed in Experiment 2, readiness potentials in this study appear to be superimposed on a prolonged positivity, which may actually be a return to baseline following termination of the contingent negative variation elicited during the warning interval (e.g., Walter et al., 1964). Because baseline displacement due to the contingent negative variation would presumably be the same preceding go and no-go stimuli, some factor other than a return to baseline must account for this go versus no-go difference (e.g., the slow positive wave associated with attentional orienting; Rohrbaugh, 1984). 
For the horizontal electro-oculogram, no effects were obtained at Epochs 1-3, but at Epochs 4-7, a difference between go and no-go trials was observed at the right eye, producing significant Site $\times$ Response interactions $(p s<.05)$. This effect was the same polarity as the go versus no-go effect at scalp sites discussed earlier, but was of lesser amplitude, consistent with the hypothesis that the effect was based on current spread from cortical loci. At forearm sites, all main effects and all interactions reached significance at all epochs except the first and ninth $(p s<.05)$. At the first epoch, there were no significant effects, and, at the ninth, significant effects were obtained for Response, Site $\times$ Response, Cued Hand $\times$ Response, and Cued Hand $\times$ Response $\times$ Site $(p s<.005)$. The pattern of means for EMG responses was very similar to that described for Experiments 2 and 3, except that amplitudes were reduced.

The failure to observe a reliable LRP on no-go trials with onset comparable in latency to that on go trials supports the asynchronous discrete coding model. Given that brief, statistically reliable LRPs have consistently been observed when preliminary information concerns a distinct stimulus attribute (Experiments 2 and 3; Osman et al., 1992), the lack of such an effect in Experiment 4 strongly suggests a qualitative difference in the transmission of partial information about single versus multiple attributes, as postulated by the asynchronous discrete coding model but not by continuous models. Furthermore, statistical analysis suggests that this is not simply a Type II error: Average no-go LRP changed significantly less from $250-500$ to $500-750$ ms poststimulus in Experiment 4 than in Experiments 2 and 3, $t(34)=2.33$, $p<.03$. Continuous models have no clear mechanism for explaining why abrupt LRP changes are larger when preliminary information is contained in a distinct stimulus attribute; precisely this effect is predicted by the asynchronous discrete coding model.

Of course, subjects might, under certain circumstances, adopt a strategy of decomposing unidimensional variation into separable codes (e.g., with practice), thereby producing a no-go LRP even with such stimuli, although our results suggest that they do not routinely do so. Furthermore, even evidence that subjects could be trained to behave this way would not necessarily support continuous models; an alternative conclusion of at least equal plausibility would be that the training had altered the subject's flexible coding scheme to decompose information about a single physical attribute into multiple psychological codes. In fact, there is evidence that such decomposition can occur when an anchor is present to support relative coding (Miller, 1983).

\section{General Discussion}

These experimental findings converge with those of Osman et al. (1992) to support the validity of the no-go LRP paradigm for assessing perceptual-motor transmission. Relying upon previous evidence that the LRP is an on-line measure of response preparation (see Coles, 1989), we have developed a paradigm in which the LRP can be used to test for response preparation resulting from partial perceptual analysis of a stimulus. The results of Experiment 2 indicate that separable attributes of a single stimulus are transmitted one at a time, in the order in which their analyses are completed. If the first attribute signals a unimanual reaction but the second indicates that the response should be withheld, response preparation begins before the second attribute has been fully processed. Once processing of the second attribute has finished, response preparation is then terminated prior to neuromuscular activation.

The possibility that this incipient response preparation on no-go trials might be generated automatically-in spite of the subject's intentions-following simultaneous transmission of both attributes was ruled out by Experiment 3 and by a related study in the Osman et al. (1992) series. Finally, Experiment 4 provided evidence against the assumption made by continuous flow (Eriksen \& Schultz, 1979) and cascade (McClelland, 1979) theories that partial results of the perceptual analysis of a single attribute are immediately transmitted to the next stage. Instead, the results support prior findings (Miller, 1982, 1983, 1987; Miller, Schäffer, \& Hackley, 1991) that interstage communication occurs in a single discrete step for any individually coded attribute. This evidence must be regarded as tentative, however, both because it is based on failure to reject a null hypothesis and because the nonsignificant effect was in the direction predicted by continuous models. Furthermore, the absence of response preparation in Experiment 4 may have been due to the difficulty of the task, which was greater than in Experiments 2 and 3, rather than to the absence of a convenient code for the information that was available early.

The results underscore the usefulness of the LRP for the study of human information processing. This utility derives from the specificity and localizability of the LRP. In comparison with many other surface potentials studied by cognitive neuroscientists (e.g., processing negativity, $\mathrm{P} 300$, and semantic N400), the LRP has both a relatively well-defined neuroanatomical origin and a relatively well-defined locus within the information-processing sequence. Thus, the LRP is likely to play a key role in interrelating neurophysiological and cognitive studies of motor preparation and mental chronometry. Our studies aid in defining the specific functional correlates of the LRP by confirming recent reports (de Jong et al., 1990; Osman et al., 1992) that this potential reflects motor processes prior to the ballistic point of no return. Because LRPs were observed in the absence of electromyographic potentials on no-go trials, it is clear that motor activation can be aborted after LRP initiation but before neuromuscular activation.

The results also have implications for the study of selective attention, in that the no-go LRP paradigm shows promise as an electrophysiological method for examining late selection (see also Coles et al., 1985). Consider the fact that on a priori grounds alone it could not be stated whether access of no-go stimuli to responses was blocked before or after the completion of sensory-perceptual analyses. Certainly, there is abundant evidence that an easy size, or spatial frequency, difference can be used as the basis for early selection (e.g., Harter \& Previc, 1978), but just how easy a discrimination has to be to mediate early selection is, as of yet, undetermined. Thus, subjects could potentially have avoided responding on no-go 
trials in Experiment 2 by filtering out or attenuating all stimuli that were of the wrong size (i.e., the no-go size). The existence of an LRP on no-go trials shows that this was not the case. Because lateralization of the readiness potential was appropriate to the shape of the stimulus, and because this lateralization terminated before neuromuscular activation, both attributes must have been perceived and transmitted to postperceptual, response-selection mechanisms. Traditionally, cognitive neuroscientists have used surface recordings to examine the mechanisms of early selection, for example, by assessing the modulation of obligatory, modality-specific, evoked potentials (reviewed by Hillyard \& Picton, 1987). Thus, use of the no-go LRP paradigm to study late selection significantly extends current methodology.

\section{References}

Amassian, V. E., Cracco, R. Q., Maccabbee, P. J., Cracco, J. B., Rudell, A., \& Eberle, L. (1989). Suppression of visual perception by magnetic coil stimulation of human occipital cortex. Electroencephalography and Clinical Neurophysiology, 74, 458-462.

Broadbent, D. E. (1958), Perception and communication. London: Pergamon Press.

Brunia, C. H. M., \& Vingerhoets, A. J. J. M. (1980). CNV and EMG preceding a plantar flexion of the foot. Biological Psychology, II, $181-191$.

Coles, M. G. H. (1989). Modern mind-brain reading: Psychophysiology, physiology, and cognition. Psychophysiology, 26, 251-269.

Coles, M. G. H., de Jong, R., Gehring, W. J., \& Gratton, G. (1988, June). Continuous versus discrete information processing: Evidence from movement-related potentials. Ninth International Conference on Event-Related Potentials of the Brain (EPICIX), Nordwick, The Netherlands.

Coles, M. G. H., Gratton, G., Bashore, T. R., Eriksen, C. W., \& Donchin, E. (1985). A psychophysiological investigation of the continuous flow model of human information processing. Journal of Experimental Psychology: Human Perception and Performance, 11, 529-553.

Coles, M. G. H., Gratton, G., \& Donchin, E. (1988). Detecting early communication: Using measures of movement-related potentials to illuminate human information processing. Biological Psychology, 26, 69-89.

Cooper, R., Osselton, J., \& Shaw, J. C. (1969). EEG technology. London: Butterworth.

de Jong, R., Coles, M. G. H., Logan, G. L., \& Gratton, G. (1990). In search of the point of no return: The control of response processes. Journal of Experimental Psychology: Human Perception and Performance, 16, 164-182.

de Jong, R., Wierda, M., Mulder, G., \& Mulder, L. J. M. (1988). Use of partial information in responding. Journal of Experimental Psychology: Human Perception and Performance, 14, 682-692.

Donders, F. C. (1969). Over de snelheid van psychische processen [On the speed of mental processes] (W. Koster, trans.). In W. G. Koster (Ed.), Attention and performance II (pp. 412-431). Amsterdam: North-Holland. (Original work published 1868)

Eriksen, C. W., \& Schultz, D. (1979). Information processing in visual search: A continuous flow conception and experimental results. Perception and Psychophysics, 25, 249-263.

Garner, W. R. (1970). The stimulus in information processing. American Psychologist, 25, 350-358.

Gemba, H., Sasaki, K., \& Tsujimoto, T. (1990). Cortical field potentials associated with hand movements triggered by warning and imperative stimuli in monkey. Neuroscience Letters, 113, 275-280.

Goodin, D. S., Aminoff, M. J., \& Shefrin, S. L. (1990). Organization of sensory discrimination and response selection in choice and nonchoice conditions: A study using cerebral evoked potentials in normal humans. Journal of Neurophysiology, 64, 1270-1281.

Gottsdanker, R., \& Shragg, G. P. (1985). Verification of Donder's subtraction method. Journal of Experimental Psychology: Human Perception and Performance, 11, 765-776.

Gratton, G., Bosco, C. M., Kramer, A. F., Coles, M. G. H., Wickens, C. D., \& Donchin, E. (1990). Event related brain potentials as indices of information extraction and response priming. Electroencephalography and Clinical Neurophysiology, 75, 419-432.

Gratton, G., Coles, M. G. H., Sirevaag, E., Eriksen, C. W., \& Donchin, E. (1988). Pre- and post-stimulus activation of response channels: A psychophysiological analysis. Journal of Experimental Psychology: Human Perception and Performance, 14, 331-344.

Green, D. M., \& Swets, J. A. (1966). Signal detection theory and psychophysics. New York: Wiley.

Grünewald, G., Grünewald-Zuberbier, E., Netz, J., Hömberg, V., \& Sander, G. (1979). Relationships between the late component of the contingent negative variation and the Bereitschaftspotential. Electroencephalography and Clinical Neurophysiology, 46, 538545.

Hackley, S. A., \& Miller, J. (1989). Lateralized readiness potentials preceding simple and complex finger movements. Psychophysiology, 26 (Suppl), 30.

Hackley, S. A., \& Miller, J. (1990). Asynchronous perceptual-motor transmission indexed by lateralized motor readiness potentials. Psychophysiology, 27 (Suppl), 36.

Hackley, S. A., Woldorff, M., \& Hillyard, S. A. (1990). Cross-modal selective attention effects on retinal, myogenic, brainstem, and cerebral evoked potentials. Psychophysiology, 27, 195-208.

Harter, M. R., \& Previc, F. H. (1978). Size-specific information channels and selective attention: Visual evoked potentials and behavioral measures. Electroencephalography and Clinical Neurophysiology, 45, 628-640.

Hillyard, S. A., \& Galambos, R. (1970). Eye movement artifact in the CNV. Electroencephalography and Clinical Neurophysiology, 18, 173-182.

Hillyard, S. A., \& Picton, T. W. (1987). Electrophysiology of cognition. In F. Plum (Ed.), Handbook of physiology: Sec. 1. The nervous system: Vol. 5. Higher Function of the Nervous System, Part 2 (pp. 519-584). Baltimore, MD: American Physiological Society.

Kolb, B., \& Whishaw, I. Q. (1990). Fundamentals of human neuropsychology. San Francisco: Freeman.

Kornhuber, H. H., \& Deecke, L. (1965). Hirnpotentialanderungen bei willkurbewegungen und passiven bewegungen des menschen: bereitschaftspotential und reafferente potentiale [Brain potential changes associated with voluntary and passive movements in humans: Readiness and reafferent potentials]. Pfluger's Archive. 284. $1-17$.

Kutas, M., \& Donchin, E. (1977). The effects of handedness, of responding hand, and of response force on the contralateral dominance of the readiness potential. In J. Desmedt (Ed.), Attention, voluntary contraction, and even-related potentials (pp. 189-210). Basel, Switzerland: Karger.

Kutas, M., \& Donchin, E. (1980). Preparation to respond as manifested by movement-related brain potentials. Brain Research, 202. 95-115.

Livingstone, M., \& Hubel, D. (1988). Segregation of form, color, movement, and depth: Anatomy, physiology, and perception. Science, 240, 740-749.

Massaro, D. W. (1975). Experimental psychology and information processing. Chicago: Rand McNally.

McClelland, J. L. (1979). On the time relations of mental processes: An examination of systems of processes in cascade. Psychological Review, 86, 287-330. 
McClelland, J. L., Rumelhart, D. E., \& the PDP Research Group. (1986). Parallel distributed processing: Explorations in the microstructure of cognition: Vol. 2. Psychological and biological models. Cambridge, MA: MIT Press.

Miller, J. (1982). Discrete versus continuous stage models of human information processing: In search of partial output. Journal of Experimental Psychology: Human Perception and Performance, 8 , 273-296.

Miller, J. (1983). Can response preparation begin before stimulus recognition finishes? Journal of Experimental Psychology: Human Perception and Performance, 9, 161-182.

Miller, J. (1985). Discrete and continuous models of divided attention. In M. I. Posner and O. S. M. Marin (Eds.), Mechanisms of attention: Attention and performance XI (pp. 513-529). Hillsdale, NJ: Erlbaum.

Miller, J. (1987). Evidence of preliminary response preparation from a divided attention task. Journal of Experimental Psychology: Human Perception and Performance, 13, 425-434.

Miller, J. (1988) Discrete and continuous models of human information processing: Theoretical distinctions and empirical results. Acta Psychologica, 67, 191-257.

Miller, J. O. (1991). Discrete versus continuous information processing: Introduction and psychophysiology. In C. H. M. Brunia, G. Mulder, \& M. N. Verbaten (Eds.), Event-related brain research: Electroencephalography and Clinical Neurophysiology (Suppl. 42, pp. 244-259). Amsterdam: Elsevier.

Miller, J., Schäffer, R., \& Hackley, S. A. (1991). Effects of preliminary information in a Go versus No-go task. Acta Psychologica, 76, 241-292.

Okada, Y. C., Williamson, S. J., \& Kaufman, L. (1982). Magnetic field of the human sensorimotor cortex. International Journal of Neuroscience, 17, 33-38.

Osman, A., Bashore, T. R., Coles, M. G. H., Donchin, E., \& Meyer, D. E. (1988). A psychophysiological study of response preparation based on partial information. Psychophysiology, 25, 426. (Abstract)

Osman, A., Bashore, T. R., Coles, M. G. H., Donchin, E., \& Meyer, D. E. (1992). On the transmission of partial information: Inferences from movement-related brain potentials. Journal of Experimental Psychology: Human Perception and Performance, 18, 217-232.

Petersen, S. E., Fox, P. T., Posner, M. I., Mintun, M., \& Raichle, M. E. (1988). Positron emission tomographic studies of the cortical anatomy of single-word processing. Nature, 331, 585-589.

Posner, M. I. (1978). Chronometric explorations of mind. Hillsdale, NJ: Erlbaum.

Riehle, A., \& Requin, J. (1989). Monkey primary motor and premotor cortex: Single-cell activity related to prior information about direction and extent of an intended movement. Journal of Neurophysiology, 61, 534-549.

Rohrbaugh, J. W. (1984). The orienting reflex: Performance and central nervous system manifestations. In R. Parasuraman \& D. R. Davies (Eds.), Varieties of attention (pp. 323-374). San Diego, CA: Academic Press.

Rohrbaugh, J. W., \& Gaillard, A. W. K. (1983). Sensory and motor aspects of the contingent negative variation. In A. W. K. Gaillard \& W. Ritter (Eds.), Tutorials in event related potential research: Endogenous components (pp. 269-310). Amsterdam: North-Holland.

Rohrbaugh, J. W., Syndulko, K., \& Lindsley, D. B. (1976). Brain components of the contingent negative variation in humans. Science, 191, 1055-1057.

Sanders, A. F. (1980). Stage analysis of reaction processes. In G. E. Stelmach \& J. Requin (Eds.), Tutorials in motor behavior (pp. 331354). Amsterdam: North-Holland.

Sanders, A. F., \& Houtmans, M. J. M. (1985). There is no central stimulus encoding during saccadic eye shifts: A case against general parallel processing notions. Acta Psychologica, 60, 323-338.

Sherrington, C. S. (1906). The integrative action of the nervous system. Cambridge, England: Charles Scribner's Sons.

Simon, J. R. (1969). Reactions toward the source of stimulation. Journal of Experimental Psychology, 81, 174-176.

Smid, H. G. O. M., Mulder, G., \& Mulder, L. J. M. (1987). The continuous flow model revisited: Perceptual and motor aspects. In R. Johnson, Jr., J. W. Rohrbaugh, \& R. Parasuraman (Eds.), Current trends in event-related potential research: Electroencephalography and Clinical Neurophysiology (Suppl. 40, pp. 270-278). Amsterdam: Elsevier.

Stein, P. S. G. (1989). Spinal cord circuits for motor pattern selection in the turtle. Annals of the New York Academy of Sciences, 563, 110.

Sternberg, S. (1969). The discovery of processing stages: Extensions of Donder's method. In W. G. Koster (Ed.), Attention and performance II (pp. 276-315). Amsterdam: North-Holland.

Sternberg, S., Monsell, S., Knoll, R. L., \& Wright, C. E. (1978). The latency and duration of rapid movement sequences: Comparisons of speech and typewriting. In G. E. Stelmach (Ed.), Information processing in motor control and learning (pp. 117-152). San Diego, CA: Academic Press.

Turvey, M. T. (1973). On peripheral and central processes in vision: Inferences from an information-processing analysis of masking with patterned stimuli. Psychological Review, 80, 1-52.

Uttal, W. R. (1973). The psychobiology of sensory coding. New York: Harper \& Row.

van der Molen, M. W., Bashore, T. R., Halliday, R. E., \& Callaway, H. (1991). Chronopsychophysiology: Mental chronometry augmented by psychophysiological time markers. In J. R. Jennings \& M. G. H. Coles (Eds.), Psychophysiology of human information processing: An integration of central and autonomic nervous system approaches (pp. 9-178). New York: Wiley.

Vaughan, H. G., Costa, L. D., \& Ritter, W. (1968). Topography of the human motor potential. Electroencephalography and Clinical Neurophysiology, 25, 1-10.

Walter, W. G., Cooper, R., Aldridge, V. J., McCallum, W. C., and Winter, A. C. (1964). Contingent negative variation: An electric sign of sensorimotor association and expectancy in the human brain. Nature, 203, 380-384.

Wijers, A. A., Mulder, G., Okita, T., Mulder, L. J. M., \& Scheffers, M. K. (1989). Attention to color: An analysis of selection, controlled search, and motor activation, using event-related potentials. Psychophysiology, 26, 89-109.

Wilson, C. L., Babb, T. L., Halgren, E., \& Crandall, P. H. (1983), Visual receptive field and response properties of neurons in human temporal lobe and visual pathways. Brain, 106, 473-502.

Received March 19, 1991

Revision received September 3, 1991 Accepted September 10, 1991 